\title{
LA TEORÍA DEL SIGNIFICADO COMO USO EN WITTGENSTENN
}

\author{
ALEJANDRO HERRERA IBÁÑEZ \\ INSTITUTO DE INVESTIGACIONES FILOSÓFICAS \\ Universidad Nacional Autónoma de México
}

El siguiente es un somero conjunto de reflexiones acerca de la tan discutida, durante muchos años, "teoría del uso", comúnmente atribuida a Wittgenstein sobre la base de algunos pasajes de las Philosopbical Investigations (PI) -aunque hay antecedentes de ella también en el Tractatus (e.g., 3.328). Particularmente sostendré que -independientemente de si Wittgenstein afirmó o no que hay identidad entre significado y uso- dicha identidad no puede darse. Sostendré también que no se puede describir teóricamente el uso de una palabra sin asignar a ésta una categoría gramatical que incluya rasgos sintácticos y semánticos, lo cual lleva a la elaboración de un conjunto de ciertos rasgos gramaticales, intensionales y extensionales, que constituyen el concepto de la palabra. La elaboración de dicho conjunto no es incompatible con el espíritu antimentalista de las PI, sino que muestra que no sólo la identificación del significado con el uso logra "sacar de la cabeza" los significados. Sostendré también que las nociones de contexto o de situación son variables para explicar lo que se ha llamado "significado del hablante", que a veces constituye una desviación del llamado "significado lingüístico" o estándar de las palabras, y este significado corresponde al uso social, o compartido por una comunidad de hablantes, en tanto que el significado del hablante es producto de un acto de habla realizado en una situación única.

Se ha afirmado que Wittgenstein jamás formuló una teoría del uso y que, por tanto, no hay nada de esto en las $P I$, especialmente una teoría en el sentido de una taxonomía de los diversos usos de las palabras. Esto es cierto, pero la pregunta interesante a propósito de ello es si Wittgenstein sostuvo que podía o que no podía haber tal tipo de teoría. Se podría argumentar que para Wittgenstein no podía haber tal teoría porque los diversos usos son para él infínitos, pero esto equivaldría a decir que tampoco puede haber teoría de los números 
naturales, por la misma razón. También se podría decir que para Wittgenstein los usos no se asemejan entre sí, lo cual hace imposible una teoría, pues afirma, por ejemplo, que describir los usos de las palabras en términos de sus referentes (decir, por ejemplo, que los signos 'a', 'b', etc., significan números) no puede eliminar la absoluta desigualdad que hay entre tales usos (PI, 10). Pero, aparte de la irrepetibilidad de cada uso, Wittgenstein parece reconocer que si bien los usos son diversos, estos pueden agruparse en diversas clases; así, hay herramientas que sirven para modificar algo (por ejemplo, el martillo, el serrucho), en tanto que otras herramientas no tienen dicha función (por ejemplo, los clavos, ia regla, el frasco de pegamento) (PI, 14). Lo mismo podría decirse de las palabras. Algunas tienen el mismo tipo de función, otras no. Parece, pues, que el problema de la elaboración de una teoría no es insalvable. La dificultad, sin embargo, es considerable, pues no tenemos "una visión clara del uso de nuestras palabras" ( $P I, 122)$, y ello se debe a que nuestra gramática no nos proporciona una "representación" clara y precisa de tales usos. Pero ello no quiere decir que no podamos elaborar una gramática que revele perspicuamente los diversos tipos de usos. Wittgenstein, sin embargo, de hecho no se propuso llevar a cabo la elaboración de tal gramática $(P I, 133)$.

Algunos comentadores han confuncido la teoría del uso con la enseñanza del uso. La enseñanza del lenguaje, o del uso de las palabras, es una especie de entrenamiento, como enseñar, por ejemplo, a un niño a lanzar y a cachar una pelota, con la resultante de que el niño lo hará sin pensar en la descripción teórica de los movimientos que ejecuta (Hunter, 391). Obviamente, este método pedagógico no elimina la posibilidad de describir teóricamente los usos:

Es bien sabido, por lo demás, que las observaciones de Wittgenstein sobre uso y significado dieron pie para el desarrollo de la teoría de los actos de habla por parte de Austin, Searle y otros. Esta teoría constituyó, según ellos, un mejoramiento de la teoría del uso (Kutschera, 162, n. 74) y es perfectamente compatible con ésta (Bogen, 200). Detrás de la teoría de los actos de habla - que constituye propiamente una teoría del uso desarrollada - está la distinción entre lenguaje, como conjunto de normas y sistemas de signos, y habla, como constituida de actos de habla particulares (Hardwick, 11, 67). Al lenguaje corresponde el significado lingüístico y al habla el significado del hablante (o, en términos de Grice, el significado oracional y el significado del emisor), distinción que, de haber sido hecha por Wittgenstein, habría aclarado el panorama haciendo el significado independiente de situaciones y usos particulares, y no determinable por éstos (Hardwick, 68).

Pero aun aceptando que no hay una teoría del uso bien desarrollada en las PI, está la afirmación de que el significado de las palabras fue definido por Wittgenstein como idéntico a su uso. Esta afirmación consta de dos partes: 
(1) Wittgenstein proporcionó una definición de 'significado', y (2) Wittgenstein dijo que significado y uso son lo mismo. Esta última afirmación tiene dos variantes: (2a) La noción de significado se reduce a la de uso, es decir, " ' $x$ ' tiene significado" significa que ' $x$ ' tiene los usos $a, b$, etc., $y(2 b)$ " ' $x$ ' significa $y$ " significa lo mismo que "el uso de ' $x$ ' es el mismo que el uso de ' $y$ "'. El pasaje que más ha sido utilizado para esta interpretación es el del famoso parágrafo 43 de las $P I$ :

Para una gran clase de casos - aunque no para todos - en que empleamos la palabra "significado", ésta puede definirse así: el significado de una palabra es su uso en el lenguaje.

Y el significado de un nombre a veces es explicado señalando a su portador.

Por un lado, en cuanto a la presencia de una definición en este parágrafo, se ha hecho notar que la forma verbal alemana erklären es traducida al inglés en el primer párrafo con el verbo definir, en tanto que la forma verbal erklärt del segundo párrafo es traducida con el verbo explicar (Hunter, 380). El verbo alemán significa dilucidar, explicar (la traducción española utiliza explicar en ambas apariciones del verbo). Se ha argumentado que la intención del texto es claramente proporcionar una definición, lo cual está lejos de ser obvio. Hay, sin embargo, otros pasajes que podrían indicar la presencia de una definición. ${ }^{1}$ En PI, 197 Wittgenstein afirma que el significado de una palabra reside en su uso. En otro lugar sugiere que la vida del signo está en el uso $(P I, 432)$. Por ejemplo, el signo ' $\longrightarrow$ ' sólo señala al ser aplicado por alguien $(P I, 454)$. Al decirle a alguien el significado de las palabras de una oración le estamos diciendo cómo aplicar las palabras de la oración y la oración misma $(P I, 175)$. Sin embargo, no parece haber en $P I$ ningún texto con una definición explícita de 'significado', y bien sabemos que Wittgenstein prefería las elucidaciones a las definiciones.

Por otro lado, se ha sostenido que lo que dice PI, 43 no es que para explicar el significado de una palabra tengamos que acudir a la explicación de su uso (Hunter, 383) - como si el significado fuese algo más, a lo que se llega a través del uso-, sino que explicar el significado es explicar el uso, de manera que decir cuál es el significado de una palabra no es decir cuál es su uso, sino que esa palabra tiene el mismo uso que otra palabra (Alston, 159; Hunter, 382). Dicha interpretación parece estar apoyada por $P I$, 30: "De manera que se podría decir: la definición ostensiva explica el uso

1 En conversación personal, Ms. Anscombe me dijo que, en efecto, tradujo crklïren con el verbo to define, porque le pareció que la intención de Wittgenstein en el texto era dar una definición. Ms. Anscombe piensa que en otros pasajes de las PI se puede ver que Wittgenstein sí quería dar una definición de "significado", pero que no hay en dicha obra ninguna definición explícita de tal palabra. 
—el significado- de la palabra...". Que el significado no proporciona el uso - dice Alston- se puede ver al reflexionar sobre el hecho de que si bien el significado de 'auténtico' es genuino, no podríamos, sin embargo, especificar el uso de 'auténtico' diciendo que el uso de 'auténtico' es genuino (Alston, 158).

A pesar de su atractiva propuesta, Alston pasa por alto que así como en el segundo párrafo de $P I, 43$ Wittgenstein dice que señalar al portador de un nombre explica a veces el significado del nombre (lo cual quiere decir que el portador no es el significado del nombre, pero tampoco excluye que sea parte del significado del nombre), en el primer párrafo dice que el uso explica el significado de una palabra, lo cual quiere decir que no todo el significado está presente en el uso.

Se ha sostenido que Wittgenstein afirma algo más específico acerca del uso, a saber, que el significado de una palabra es su uso en un juego de lenguaje. Dice, por ejemplo, que decir que un nombre ha dejado de ser significativo es lo mismo que decir que ese nombre ya no tiene ningún uso en el juego de lenguaje al que pertenecía $(P I, 41)$. Si se ve un juego de lenguaje como un sistema, no tener una función en el sistema es no ser significativo en el sistema. Sin embargo, no está claro que Wittgenstein haya visto el lenguaje como una colección de sistemas discretos, o sea, como una colección de juegos de lenguaje. Parece, más bien, haberlo visto como una masa nebulosa rodeada por juegos de lenguaje técnicos más o menos claros (Bogen, 204).

El segundo párrafo de $P I, 43$ afirma que la definición ostensiva de una palabra a veces dilucida o explica su uso, y en $P I, 30$ Wittgenstein afirma que esto sucede "cuando ya está claro qué papel debe desempeñar en general la palabra en el lenguaje". Esta afirmación parece indicar que el significado está dado principalmente por un conjunto de reglas de uso. Sabemos qué decir cuando el uso de una palabra está claramente prescrito $(P I, 142)$, o sea, cuando sus reglas de uso son claras. Y cuando usamos (correctamente) una palabra, estamos obedeciendo una regla (de uso) (PI, 199): "Entender una oración significa entender un lenguaje. Entender un lenguaje significa dominar una técnica" (id.), es decir, entender un lenguaje es ajustarse a sus reglas de uso, y estas reglas determinan el significado de las palabras. Así, por ejemplo, señala Wittgenstein que la palabra 'no' carece de significado si no se le asocian reglas de uso, y si se le asignan otras reglas adquiere otro significado (o no adquiere ninguno) $(P I, 147)$. El ejemplo es interesante, porque Wittgenstein explica su afirmación utilizando una partícula sincategoremática que no va más allá de su función sintáctica. En el caso de una palabra categoremática las reglas de uso deberán señalar rasgos adicionales, específicamente rasgos semánticos. La descripción del uso es, pues, algo complejo. Por ejemplo, en $P I, 26$ sugiere Wittgenstein que a quien diga que nombrar algo es un preparativo para usar una palabra, se le puede preguntar: ¿de qué es un preparativo? 
Es decir, el nombrar es ya un tipo de uso de una palabra. Así, podemos decir que 'tigre' se usa como nombre en tanto que 'feliz' se usa como atributo. Pero esto significa que parte al menos de la descripción del uso de una palabra consiste en decir a qué categoría gramatical pertenece. Obviamente, esto no es suficiente para dar el significado de 'tigre' o de 'feliz'.

Todo lo anterior sugiere que si el uso, o las reglas de uso, no agotan el significado de una palabra, entonces Wittgenstein no estaba proponiendo una identidad rigurosa entre significado y uso, sino más bien estaba haciendo una propuesta metodológica para acercarse de una manera segura al significado de las palabras. Desde el inicio de las PI dice Wittgenstein: “¿Pero cuál es el significado de la palabra 'cinco'. - No se habla aquí en absoluto de tal cosa; sólo de cómo se usa la palabra "cinco'" ( $P I, 1)$. Esta observación no es una propuesta para sustituir siempre la pregunta sobre el significado por la pregunta sobre el uso. Se trata más bien de una recomendación práctica: "Mira la oración como un instrumento, y su sentido como su empleo" (PI, 421), o también: "Digamos que el significado de una pieza es su papel en el juego" $(P I, 563)$. "No preguntes por el significado; pregunta por el uso". Esta regla práctica sirve para enseñarnos el significado y sirve como criterio de la comprensión del significado de una palabra: "Deja que el uso te enseñe el significado" (PI, p. 212). Para saber si alguien entendió una definición como "'Este número se llama dos' hay que observar cómo usa la palabra definida" (PI, 29). Así, el uso es un criterio o medida de la comprensión del significado de una palabra, pero no es el significado mismo. Al decirnos en otro lugar que dejemos que el uso de las palabras nos enseñe el significado de éstas, añade: "De manera semejante puede uno decir en matemáticas: deja que la prueba te enseñe lo que se estaba probando" (PI, p. 220). Siguiendo el símil, así como la prueba no se identifica con lo probado, así el uso no se identifica con el significado. Algunos filósofos han llegado a pensar que la recomendación wittgensteiniana va dirigida a filósofos, pero no a lexicógrafos o traductores, y que se trata, en ciertos casos, de examinar las palabras funcionando, o sea, de pasar de su poder permanente de compra cuando están en el banco a la acción concreta del mercado realizada con ellas ayer en la mañana (Ryle, 114). La propuesta de Wittgenstein es que veamos la palabra y su significado como el dinero y su uso, y no como el dinero y la vaca que con él se puede comprar $(P I, 120)$. De esta manera queda descartada la visión denotativista del significado.

¿Y qué hay de la visión connotativa del significado, o sea, del significado como un conjunto de características? Aquí Wittgenstein es muy cauteloso. En $P I, 138$ llega a aceptar que una cosa es el uso, el cual se extiende en el tiempo, y otra cosa es entender el significado de una palabra al oírla o decirla. En este 
caso la captamos "de golpe". Parece seguirse que entender el significado no es entender el uso. La comprensión es instantánea, pero todo el uso de una palabra no puede presentarse ante la mente con ese tipo de comprensión (PI, $139-40,191)$. Le tomará varios parágrafos a Wittgenstein salir del atolladero y llegar a la conclusión de que no hay nada sorprendente ni raro en este problema. La rareza surge cuando pensamos que captar el uso es captar el desarrollo futuro. Comprender el significado de una palabra es comprender su uso, pero ello no quiere decir que los usos futuros de la palabra deban estar presentes en dicha comprensión $(P I, 197)$. Queda, sin embargo, la impresión de que Wittgenstein ha aceptado que hay algo más que la captación del uso en la captación del significado. En otro parágrafo parece quedar abierta la posibilidad de aceptar que dos palabras con el mismo uso pueden estar asociadas a ideas diferentes $(P I, 556)$. Hacia lo que quiero apuntar es que si la fórmula "significado-uso" es una recomendación metodológica, entonces es posible que Wittgenstein estuviese dispuesto a aceptar que el significado tiene otros ingredientes, siempre y cuando la nueva formulación no tenga ni tintes denotativistas ni tintes mentalistas. Pero si la fórmula "significado-uso" es una tesis fuerte, ésta parece ser falsa a menos que la noción de uso se amplíe de manera tal que las distintas características asociadas a una palabra, digamos, ambigua, constituyan los distintos usos de la palabra. Se ha argumentado que uno puede conocer el uso de una palabra sin coriocer su significado, y que también se puede saber el significado de una palabra sin saber cómo se usa (Pitcher, citado en Kutschera, 115). Podría, por ejemplo, como hablante nativo del español con ciertas nociones de inglés, saber el significado de "God bless you" ("Dios te bendiga") sin saber usarlo después de que alguien estornuda. Podría, por otro lado, después de averiguar el uso de "God bless you" y de enterarme de que "Gesundheit" tiene el mismo uso, saber usar "Gesundheit" sin saber que significa "salud" y creyendo que significa "Dios te benciiga". También se ha argumentado que el uso comendativo de 'bueno' no nos da el significado de la palabra (Hunter, 375). Además, el uso comendativo es el mismo para 'hombre bueno', i. e., moralmente virtuoso, que para 'manzana buena', expresión que tiene otro significado (id., 376). También se ha argumentado que la noción de uso presupone la noción de significado. La noción de uso es más amplia que la de significado, puesto que cubre muchas otras cosas, pero es el significado de la palabra el que regula sus diversos usos y no viceversa (Findlay, 118).

Dar el significado de una palabra es, a grandes rasgos, decir qué connota y denota dicha palabra (Findlay, 119). Por raro que suene, me inclino a pensar que esta visión del significado no es incompatible con algunas de las tesis de Wittgenstein, especialmente la que afirma que el significado no es mental. A Wittgenstein le interesa que si en ruso se dice "piedra roja" en lugar de "la piedra es roja" no se sostenga que el hablante nativo está suplementando su 
oración mentalmente con la cópula "es" $(P I, 20)$. La experiencia del significado - nos dice - no tiene que ver con la experiencia de una imagen mental (PI, pp. 175-6). No hay una tercera entidad mental que explique la identidad de usos o significados. "iLosa!" y "Tráeme una losa" tienen el mismo sentido, y éste no tiene que expresarse con otra oración (PI, 20). Hacerlo sería postular representaciones ocultas del significado, y Wittgenstein sostiene que "nada está oculto" (PI, 435). Para él, la representación está en el uso. Sin embargo, no veo el porqué de la negativa a aceptar la postulación de entidades teóricas, con poder explicativo, como sucede normalmente en las ciencias. Postular una estructura profunda de dos palabras u oraciones con el mismo sentido no es necesariamente postular una imagen presente en la mente de los hablantes, sino un constructo teórico que no tiene por qué aparecer en nuestras mentes cuando comprendemos el significado de una palabra. Podrán aparecer partes del constructo, pero no necesariamente todo el constructo. Igualmente es posible dar descripciones teóricas de los usos de las palabras sin comprometerse con que dichas descripciones estén presentes en las mentes de los hablantes. Por lo demás, hay indicios de que Wittgenstein no excluye la posibilidad de representar los usos. Sólo necesitamos una gramática perspicua $(P I, 122)$ que pueda lidiar con la gran diversidad de usos (Bogen, 214). La lingüística es, después de todo, aún muy joven. Wittgenstein mismo proporciona - aun con sus reticencias antiteóricas- un ejemplo de lo que podría ser un análisis de los usos de una palabra. Al analizar el significado del verbo entender, encuentra en él dos usos: en uno de ellos se reemplaza lo que se quiere entender por algo con un uso similar, y dice que a estos corresponde un pensamiento común; en el otro uso, entender es captar lo que de singular tiene una palabra u oración (como cuando se lee poesía) (PI, 531). Se pregunta Wittgenstein si entender tiene dos significados (puesto que tiene dos usos) y se responde que los dos usos constituyen el significado de entender, constituyendo así -dice- "mi concepto de entender", y agrega: "pues quiero aplicar la palabra 'entender' a todo esto" (PI, 532). Este ejercicio wittgensteiniano apunta a favor de la idea de que el significado tiene que ver con un conjunto de usos (o de reglas de uso) al que va asociado un concepto en la mente del hablante. El conjunto es, sin embargo, un constructo teórico (ver Kutschera, 142) que recoge tipos de uso. Pero hay que distinguir entre el uso social de una palabra, el cual está asociado a un significado lingüístico más o menos estable, y el uso (o usanza) de una palabra en un contexto o situación determinados, el significado del hablante, que puede tener varias desviaciones del significado original. Mostrar más en detalle la compatibilidad de esta propuesta con la propuesta wittgensteiniana rebasa indudablemente los límites de este trabajo. 


\section{BIBLIOGRAFIA}

Alsron, William P., "Meaning and Use", en Parkinson.

Bogen, James, Wittgenstein's Philosoply of Language, Londres, Routledge \& Kegan Paul, 1972.

Findlay, J. N., "Use, Usage and Meaning (2)", en Parkinson.

Hardwick, Charles S., Langurge Learning in Wittgenstein's Later Pbilosophy, La HayaParís, Mouton, 1971.

Hunter, "Wittgenstein on Meaning and Use", en Klemke (ed.), Essays on Wittgenstein, Urbana, Ill., University of Illinois Press, 1974.

Kutschera, Franz von, Philosophy of Language, Dordrecht, Reidel, 1975.

Parkinson, G. H. R. (ed.), The Theory of Meaning, Oxford, Oxford University Press, 1968.

Ryle, Gilbert, "Use, Usage and Meaning (1)", en Parkinson.

Wittgenstein, Ludwig, Pbilosophical Investigations, trad. por G. E. M. Anscombe, Oxford, Basil Blackwell, 1968. [Traducción española de Alfonso García Suárez y Ulises Moulines, Barcelona, Instituto de Investigaciones Filosóficas-UNAM/Editorial Crítica, 1988.] 